University of New Hampshire

University of New Hampshire Scholars' Repository

Physics Scholarship

Physics

7-14-2011

\title{
New measurements of total ionizing dose in the lunar environment
}

\author{
J. E. Mazur \\ W. R. Crain \\ M. D. Looper \\ D. J. Mabry \\ J. B. Blake
}

See next page for additional authors

Follow this and additional works at: https://scholars.unh.edu/physics_facpub

Part of the Physics Commons

\section{Recommended Citation}

Mazur, J. E., W. R. Crain, M. D. Looper, D. J. Mabry, J. B. Blake, A. W. Case, M. J. Golightly, J. C. Kasper, and H. E. Spence (2011), New measurements of total ionizing dose in the lunar environment, Space Weather, 9, S07002, doi:10.1029/2010SW000641.

This Article is brought to you for free and open access by the Physics at University of New Hampshire Scholars' Repository. It has been accepted for inclusion in Physics Scholarship by an authorized administrator of University of New Hampshire Scholars' Repository. For more information, please contact Scholarly.Communication@unh.edu. 


\section{Authors}

J. E. Mazur, W. R. Crain, M. D. Looper, D. J. Mabry, J. B. Blake, A. W. Case, M. J. Golightly, J. C. Kasper, and Harlan E. Spence 


\title{
New measurements of total ionizing dose in the lunar environment
}

\author{
J. E. Mazur, ${ }^{1}$ W. R. Crain, ${ }^{2}$ M. D. Looper, ${ }^{2}$ D. J. Mabry, ${ }^{2}$ J. B. Blake, ${ }^{2}$ A. W. Case, ${ }^{3}$ \\ M. J. Golightly, ${ }^{4}$ J. C. Kasper, ${ }^{3}$ and H. E. Spence ${ }^{4}$ \\ Received 13 November 2010; revised 21 March 2011; accepted 15 April 2011; published 14 July 2011.
}

[1] We report new measurements of solar minimum ionizing radiation dose at the Moon onboard the Lunar Reconnaissance Orbiter (LRO) from June 2009 through May 2010. The Cosmic Ray Telescope for the Effects of Radiation (CRaTER) instrument on LRO houses a compact and highly precise microdosimeter whose design allows measurements of dose rates below 1 micro-Rad per second in silicon achieved with minimal resources ( $20 \mathrm{~g}, \sim 250$ milliwatts, and $\sim 3 \mathrm{bits} / \mathrm{second})$. We envision the use of such a small yet accurate dosimeter in many future spaceflight applications where volume, mass, and power are highly constrained. As this was the first operation of the microdosimeter in a space environment, the goal of this study is to verify its response by using simultaneous measurements of the galactic cosmic ray ionizing environment at LRO, at L1, and with other concurrent dosimeter measurements and model predictions. The microdosimeter measured the same short timescale modulations in the galactic cosmic rays as the other independent measurements, thus verifying its response to a known source of minimum-ionizing particles. The total dose for the LRO mission over the first 333 days was only 12.2 Rads behind $~ 130$ mils of aluminum because of the delayed rise of solar activity in solar cycle 24 and the corresponding lack of intense solar energetic particle events. The dose rate in a $50 \mathrm{~km}$ lunar orbit was about 30 percent lower than the interplanetary rate, as one would expect from lunar obstruction of the visible sky.

Citation: Mazur, J. E., W. R. Crain, M. D. Looper, D. J. Mabry, J. B. Blake, A. W. Case, M. J. Golightly, J. C. Kasper, and H. E. Spence (2011), New measurements of total ionizing dose in the lunar environment, Space Weather, 9, S07002, doi:10.1029/2010SW000641.

\section{Introduction}

[2] The total ionizing dose (TID) hazard originates from the space environment and includes contributions from charged particles (electrons, ions, and secondary charged particles such as muons and pions), neutrons that undergo nuclear collisions to produce charged secondaries, and primary photons from the environment and electron bremsstrahlung. Whatever the primary origin, the effect of concern is the deposition of energy in the form of free charge within materials. The free charge in turn can affect surface chemistry, microelectronic device operation, and material properties [e.g., Stuckey and Meshishnek, 2003; Pease et al., 2009]. TID is also a concern for long-term human exploration of space [e.g., Cucinotta et al., 2005, and

\footnotetext{
${ }^{1}$ Aerospace Corporation, Chantilly, Virginia, USA.

${ }^{2}$ Aerospace Corporation, El Segundo, California, USA.

${ }^{3}$ Harvard-Smithsonian Center for Astrophysics, Cambridge, Massachusetts, USA.

${ }^{4}$ Institute for the Study of Earth, Oceans, and Space, University of New Hampshire, Durham, New Hampshire, USA.
}

references therein] because there are no analogs for human exposure to TID from the space environment other than that already acquired on human space missions.

[3] One assesses the TID impacts through testing of electronic parts, testing of spacecraft materials using photon sources and particle accelerators, modeling of the space environment in the mission orbit, and ray tracing to model the distribution of mass and hence the TID reduction (or increase) at the location of interest inside the space vehicle.

[4] An independent method of addressing the TID hazard is to measure it directly on-orbit using a calibrated instrument. There are many examples of dosimetry using standard techniques of charge collection in a known detector volume. The principal benefit from a direct measurement is the reduction of uncertainty compared to that from the process of measuring the external environment, including all the particle and photon inputs, and modeling the transport of that environment and its interaction with materials. Hence, the direct measurement of TID has uncertainty that originates primarily from the dosimeter's ability to accurately capture the liberated 
charge in a test mass and to accurately report that result. Of course, one disadvantage is that the results are not known for a particular mission until the mission has flown.

[5] Direct TID measurements typically rely on telemetered data so that a return of the dosimeter is not required, as it is in the case of passive devices such as thermoluminescent detectors. Current examples of active dosimetry include the dosimeters on several GPS vehicles [Cayton et al., 1998] and in highly elliptical orbit [Blake et al., 1997]. These devices also provide information about the near real-time state of the ionizing radiation environment for the mission, as well as a history of the environment that one can use for scientific investigations of the charged particle inputs. For example, Fennell et al. [2003] used the long-term histories of the TID in highly elliptical orbits to study the effects of electron sources and losses at different altitudes in the outer radiation belt. Thus a relatively simple dosimeter can provide quantitative information about physical processes with relatively low requirements for telemetry and other spacecraft resources compared to comprehensive science instruments $\left[\mathrm{O}^{\prime}\right.$ Brien et al., 2008].

[6] We discuss here the initial results from a microdosimeter that was included as part of a scientific instrument on the NASA Lunar Reconnaissance Orbiter (LRO). Since this is the first use of the microdosimeter in a space environment, our goal here is to benchmark its performance using the first year of the LRO mission. We show the specifics of this new dosimeter design and implementation on LRO, its response during various orbital phases of the LRO mission, and new results related to the ionizing radiation environment within $\sim 200 \mathrm{~km}$ of the lunar surface.

\section{Instrumentation}

[7] The LRO mission objective is to investigate the lunar environment to prepare for and support future manned exploration of the Moon [Vondrak et al., 2010]. The seveninstrument payload was selected to meet the NASA Exploration Systems Missions Directorate objectives that included characterization of lunar radiation hazards and temperature mapping in permanently shadowed regions (cf. Vondrak et al. [2010] for more details of the LRO payload and mission objectives). LRO was launched on 18 June 2009 and on 15 September 2009 it entered the prime mission orbit to map the lunar surface and address the other mission objectives from a $50 \mathrm{~km}$ altitude.

[8] One of the LRO science instruments addresses the ionizing radiation hazard: the Cosmic Ray Telescope for the Effects of Radiation (CRaTER). Spence et al. [2010] discussed the instrument design and objectives. The CRaTER instrument uses energy deposition within silicon solid state detectors and inert tissue-equivalent plastic, backed by a detailed calibration and characterization database, to accurately determine the linear energy transfer spectrum at LRO. The dosimeter that is the sub- ject of this study is housed within the CRaTER instrument and its data included in the CRaTER telemetry. We refer to specific CRaTER results in this paper only as they relate to the dosimeter characterization and performance. The work by Case et al. [2010] is an example of detailed CRaTER science that is ongoing as the LRO mission continues into a NASA Science Mission Directorate phase.

[9] The microdosimeter that is part of the CRaTER investigation is the latest embodiment of a design that originated in the 1960s [Freden et al., 1965]. For the LRO mission and future applications, we have miniaturized the electronics to minimize the resource impacts and allow for more targeted placement of the dosimeter within the spacecraft. We also made the charge collection more accurate as discussed below.

[10] Figure 1a shows the microdosimeter internal components. The resource and implementation table below provides more detail on the device size, and the U.S. dime in Figure 1a is to scale. We removed the 10 mil thick Kovar lid from the dosimeter for the photograph. There were two design objectives that allowed for the small scale of the device.

[11] The first objective was to place the bulk of the signal processing in a custom CMOS Application Specific Integrated Circuit (ASIC). The ASIC development allowed the dosimeter design to be much smaller than previous applications that used discrete components and on the order of $10 \mathrm{~cm}^{2}$ of printed circuit board area. Another prime design element that allows for such a small scale is the relatively high resistivity of the silicon detector (on the order of $10^{5} \mathrm{ohm}-\mathrm{cm}$ ) so that a high-voltage power supply is not required to fully deplete the silicon detector; instead, the silicon fully depletes with typical spacecraft voltages. Micron Semiconductor Ltd. manufactured the detector to the size and resistivity requirements. Teledyne Microelectronics Technologies manufactured the dosimeter shown in Figure 1 (http://www.teledynemicro.com/ space/space_micro_dosimeter.asp).

[12] Figure $1 \mathrm{~b}$ charts the signal flow and processing. First, the ionizing radiation field liberates free charge within the silicon detector. This is the direct equivalent of TID. The ASIC amplifies current pulses and successive deposits (above $100 \mathrm{keV}$ ) are integrated until the integrated total charge equals a single dose quantum, in this case 13.6 micro-Rads. The ASIC integrates the signal prior to the threshold detection in order to improve the accuracy of the charge collection. Once the quantum is reached, the counters increment and the TID is output as a proportional voltage at the four device outputs. We designed the analog dose outputs to be compatible with common spacecraft analog housekeeping systems. Table 1 summarizes the specific design characteristics of the microdosimeter including the physical parameters, the electrical requirements, and the analog voltage outputs. There is also a test input to the device that allows for laboratory pulser stimulation of the ASIC independent of the silicon detector for problem diagnosis and calibration. The 

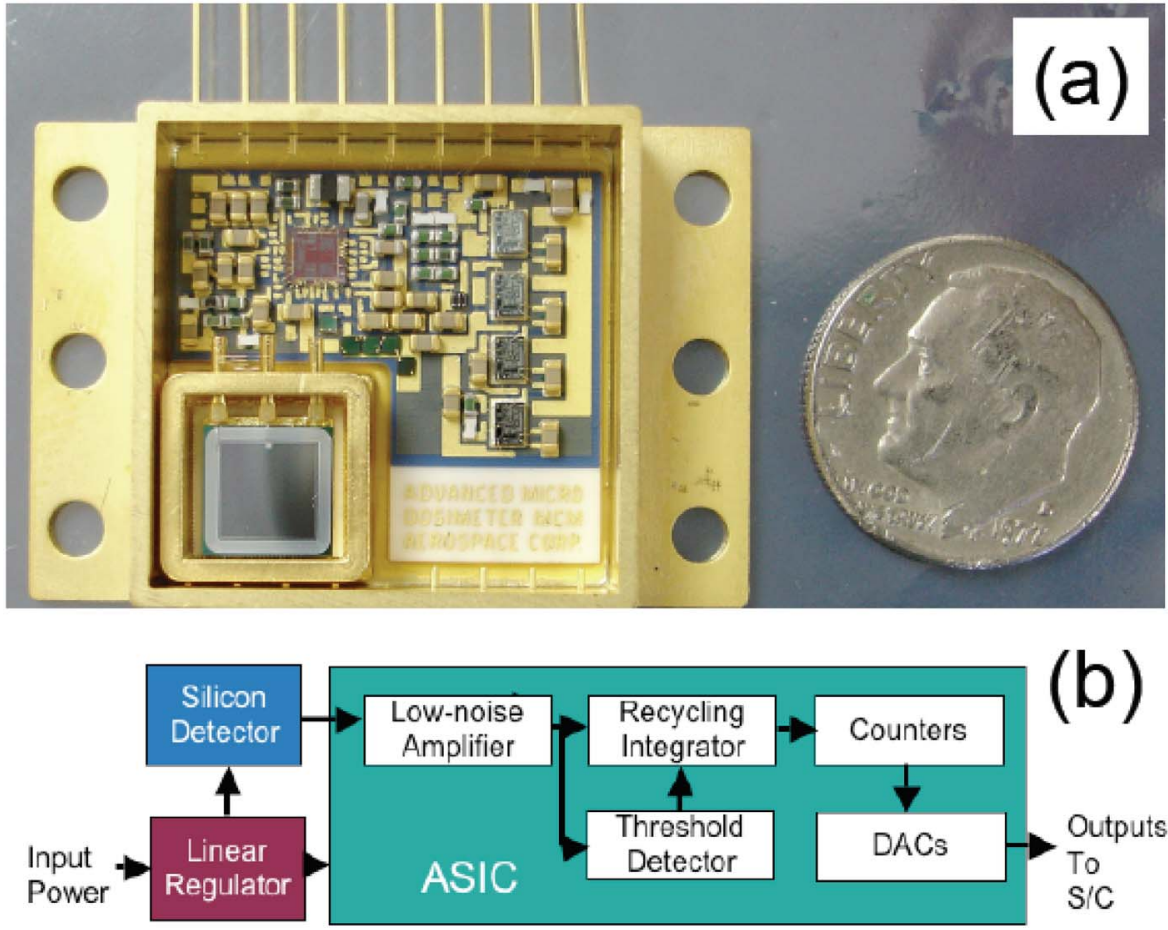

Figure 1. (a) Microdosimeter components (device lid has been removed for this photograph). The gray square at lower left is the silicon detector with $5 \mathrm{~mm} \times 5 \mathrm{~mm}$ active area. (b) Microdosimeter signal flow.

equivalent LET (linear energy transfer) range for the CRaTER device is $\sim 0.4$ to $60 \mathrm{keV} /$ micron.

[13] The analog-to-digital conversion values of the accumulated dose continually increment by the steps listed in Table 1; the dosimeter holds these values and increments them, rolling them over once the limits are reached, as long as the device is powered. Thus for typical applications, the bookkeeping of when the different ranges roll over, the tracking of when power cycles occur, and the calculation of dose rate are all performed during ground-based processing of the data.

[14] The charge collection is the principal determinant of the device accuracy. Testing of the integrator with a calibrated pulser resulted in a rectangular efficiency curve (not shown) with a typical accuracy of $+/-20 \%$. The primary source of variability among devices with this specific design is random amplifier offsets inside the ASIC. For most applications this TID accuracy is sufficient. In comparison with the ground-based modeling technique, some environments are typically thought to be known no better than a factor of two, and that is independent of the additional and usually unquantified uncertainties in propagating that environment through a specific system design.

[15] We have tested the microdosimeter design for single-event effects and total dose hardness, and we studied dosimeter performance with gamma-ray sources and a $50 \mathrm{MeV}$ proton beam at the Lawrence Berkeley Laboratory 88 inch cyclotron. The dosimeter has no total dose degradation up to at least $100 \mathrm{kRad}$, shows no latchup, and is able to accurately measure a $20 \mathrm{kRad}$ proton dose delivered in a time period of only $30 \mathrm{~min}$.

[16] We housed a single microdosimeter inside the CRaTER instrument as an opportunity to provide an independent measurement of the TID environment on LRO and also as a way to gauge the microdosimeter

Table 1. LRO/CRaTER Microdosimeter Resources and Performance Specifications

\begin{tabular}{lcc}
\hline \multicolumn{1}{c}{ Parameter } & Value(s) & Unit \\
\hline Total mass $^{\text {a }}$ & 20 & $\mathrm{~g}$ \\
Power $^{\mathrm{a}}$ & 0.28 & Watts \\
Dosimeter outer dimensions $^{\text {Silicon detector dimensions }}{ }^{\mathrm{b}}$ & $3.5 \times 2.5 \times 0.45$ & $\mathrm{~cm}$ \\
Detector electronic threshold $^{\text {Maximum energy deposit }}$ & $0.5 \times 0.5 \times 2.5 \mathrm{e}-02$ & $\mathrm{~cm}$ \\
LET range & 100 & $\mathrm{keV}$ \\
DAC low range & $0.4-60$ & $\mathrm{MeV}$ \\
DAC low step size & $0-3.5$ & $\mathrm{keV} / \mu$ \\
DAC medium range & 13.6 & $\mathrm{mRads}$ \\
DAC medium step size & $0-0.88$ & $\mu$ Rads \\
DAC high range & 3.5 & Rads \\
DAC high step size & $0-233$ & $\mathrm{mRads}$ \\
DAC logarithmic range & 0.88 & Rads \\
Dose rate & $0-68$ & Rads \\
\hline
\end{tabular}

${ }^{\text {a}}$ Power at $28 \mathrm{~V}$ input.

${ }^{\mathrm{b}}$ Detector active area is square with thickness of $250 \mu$.

${ }^{c}$ We did not use the logarithmic range for the CRaTER application. 

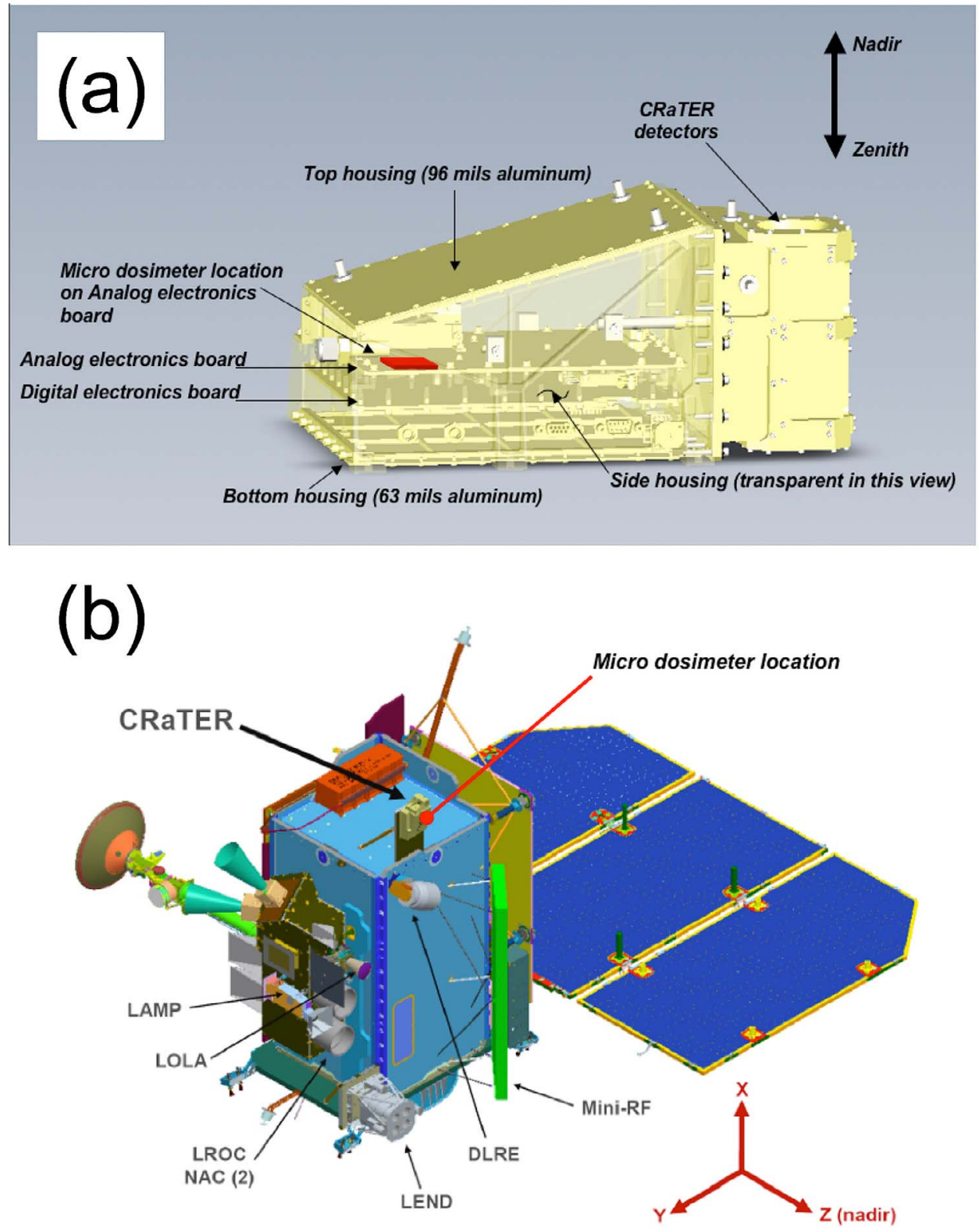

Figure 2. (a) Location of the microdosimeter within the CRaTER instrument. (b) Orientation of $\mathrm{CRaTER} /$ microdosimeter on the LRO vehicle. In this coordinate system the $\mathrm{z}$ axis normally points toward the surface of the moon.

device performance with simultaneous measurements from a well-calibrated scientific instrument. Figure $2 a$ is a diagram of the microdosimeter location within CRaTER. The device is mounted to one side of the CRaTER analog electronics printed circuit board. With this location, the planar silicon detector inside the dosimeter is coplanar with the silicon detectors in the CRaTER telescope.

[17] Figure $2 \mathrm{~b}$ places the CRaTER and microdosimeter locations in the context of the LRO vehicle and its nominal attitude at the Moon. Being within the CRaTER housing means that the CRaTER structure and internal circuit boards were the primary obstructions for the microdosimeter; very little of the detector geometry factor included the spacecraft itself. Note that in the nominal LRO mapping attitude, one side of the dosimeter views the lunar surface through a minimum of approximately $0.89 \mathrm{~g} / \mathrm{cm}^{2}$ or equivalently 131 mils of aluminum while the other side views space through $2.28 \mathrm{~g} / \mathrm{cm}^{2}$ or $332 \mathrm{mils}$ aluminum. We have not performed a detailed ray tracing of the mass distributions because the dominant environ- 
ment for this study consisted of minimum ionizing galactic cosmic rays, where the shielding afforded by either side is minor and approximately equivalent. We note that the incidental shielding corresponds to penetration ranges of protons with incident energies of $\sim 24$ to $38 \mathrm{MeV}$, for reference in future studies of the dosimeter's response to primary particles in solar energetic particle events.

[18] Table 1 also lists the data acquisition parameters for the microdosimeter. The most important aspect for this study is that the three linear digital-to-analog conversion (DAC) voltages were sampled and telemetered at a $16 \mathrm{~s}$ cadence as part of the CRaTER instrument housekeeping data packets. Housekeeping data packets and thus microdosimeter voltages were in the data stream whenever the instrument received the $28 \mathrm{~V}$ power from the LRO vehicle. Several power cycles that occurred during the first 11 months of the mission reset the microdosimeter accumulators to zero as designed (see results section below).

[19] We obtained data from the microdosimeter during CRaTER instrument environmental testing and calibration and during LRO spacecraft environmental testing. There was never any signature of electromagnetic interference that might contribute to noise counts in the detector. We did not observe any noise during high temperature instrument tests (above $20^{\circ} \mathrm{C}$ ) where one might expect a higher leakage current and thus noise contributions to the TID counts. For reference, the daily averaged on-orbit temperature ranged between $-15^{\circ} \mathrm{C}$ and $8^{\circ} \mathrm{C}$ for this study period.

\section{Observations and Dose Analysis}

[20] For this study we used the LRO/CRaTER measurements acquired from 20 June 2009 to 31 May 2010. There were 8 complete days excluded out of the 333 of this study (approximately 2\%) when CRaTER was powered off for all or part of the day during spacecraft safe attitude intervals or other significant spacecraft events. Thus, the results below are a nearly complete record of the TID and dose rate for the LRO mission that covers most of the interplanetary transit to lunar orbit, the commissioning phase, and the first 245 days of the mission orbit at nominal $50 \mathrm{~km}$ altitude. We first demonstrate the day-today operation of the microdosimeter, and we then use the accumulated dose measurements to calculate the dose rate whose properties we explore as a function of time and altitude above the Moon.

\subsection{Microdosimeter Operation}

[21] We show in Figure 3 a typical day of microdosimeter operation on LRO. These were 5 min averages of all dosimeter output values, sufficient for these displays and the analysis below because the TID rate due to galactic cosmic ray (GCR) changed slowly on the timescale of several hours. Figure 3 (top) shows the highestresolution DAC output as it accumulated dose in 13.6 micro-Rad steps during the day. Note that the dose rate was not constant in this interval, as there were several intervals when it took less or more time to increase the accumulator by one step. There were 11 instances in Figure 3 when the low-range/high-resolution accumulator reached the maximum value of 3.5 milli-Rads, whereupon the midrange accumulator increased by 3.5 milli-Rads; this counter rolled over after 14:20 UT after reaching the maximum range of 0.88 Rads. Finally, on this day the highrange accumulator incremented by one step (0.88 Rads) near 14:20 UT.

\subsection{TID for First 333 Days of LRO Mission}

[22] Figure 4 shows the result of adding the microdosimeter accumulators to obtain a TID history of the mission through 31 May 2010. The TID value at the end of the time series is 12.0 Rads. The longest interval without measurements (approximately 6 days in February 2010) constituted a loss of approximately 0.2 Rads from the measured TID shown in Figure 4. Therefore the dose for the mission through 31 May 2010 is 12.2 Rads. During an interval when the slowly varying galactic cosmic rays dominate the TID, using a constant dose rate in order to estimate the missing TID from such gaps in the record is a reasonable approach. Another feature of the TID history was the subtle change in slope of the dose accumulation that occurred when LRO entered the prime mission orbit in September 2009. One can derive an approximate average dose rate from Figure 4 by dividing the TID by the total time; this is about 4 micro-Rads/sec, which agrees with the dose rate calculated versus time in the next section.

\subsection{Dose Rate}

[23] It was straightforward to calculate a dose rate from the low-range DAC output in ground-based processing simply by differencing the low-range outputs (sampled every $16 \mathrm{~s}$ ). Because the dose rate was low for this study period, this meant that often the successive low-range values were identical, leading to a zero calculated rate for many $16 \mathrm{~s}$ intervals. However, these values were the best estimates of the time dependence of the dose rate; that is, often we measured a constant dose rate within the time resolution of the measurement.

[24] Given the DAC sampling rate and the relatively low dose rate due to GCR, we show in Figures 5, 6, and 7 below dose rates averaged over longer time periods appropriate for other investigations of its response. Figure 5 shows the $60 \mathrm{~min}$ averaged dose rate during the approach to the Moon, lunar orbit insertion, and the initially highly eccentric orbits of LRO. While LRO was far from the Moon, the measured dose rate varied by $\sim 0.1$ micro-Rads/sec, less than a $10 \%$ variability. Once in an eccentric lunar orbit, Figure 5 shows that the altitude ordered the dose rate in that the minimum rate usually occurred at minimum altitude. During the commissioning phase of the mission in Figure 5, a vector normal to the microdosimeter detector intersected the lunar surface at periselene, thus maximizing the Moon's obscuration of nearly half of the detector's field of view. Thus, even in 

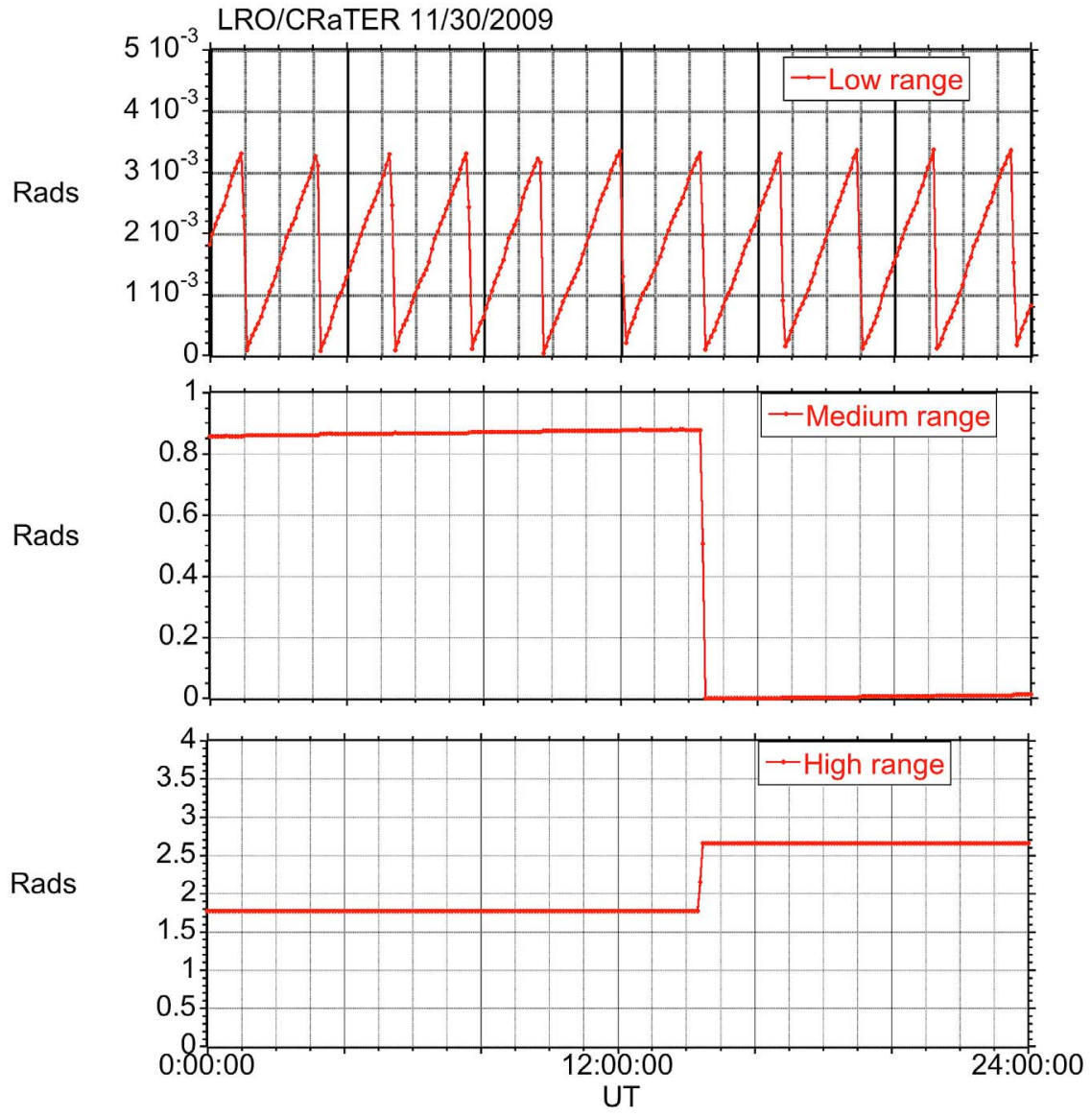

Figure 3. Example of the microdosimeter dose ranges for a typical day of the LRO mission (30 November 2009). These were $5 \mathrm{~min}$ averages of the $16 \mathrm{~Hz}$ DAC readouts. The dose accumulators rolled over at 13.5 micro-Rads and 0.88 Rads for the low and medium ranges, respectively. Shown is the 0.88 Rad step size of the high range. The TID shown in this day originated from galactic cosmic rays.

this relatively low level of analysis one can see the Moon's GCR shadow (e.g., Lin [1968] and discussion of GCR response below).

[25] Figure 6 illustrates the same altitude effect but over the entire study period, demonstrating that the largest change observed in dose rate occurred when LRO went into lunar orbit. Here we used daily averages of the dose rate without any restriction on LRO attitude. Once in lunar orbit, the entry to the $50 \mathrm{~km}$ mission orbit in September 2009 as well as time variations in the GCR environment led to changes on the order of 10 percent.

[26] In Figure 7 we map dose rate as a function of LRO altitude instead of time. Here we see again that the average dose rate decreased with decreasing altitude, with more variability visible at $\sim 50$ and $210 \mathrm{~km}$ because of the longer residence times at those altitudes. Because of the LRO orbit it was not possible to correct the altitude dependence for the concurrent time dependence of the TID environment. However, we note that the approximate
$30 \%$ decrease from interplanetary dose rate to the rate at $50 \mathrm{~km}$ in Figure 8 is consistent with the lunar obscuration of the sky [e.g., Lin, 1968].

\subsection{Response to GCR}

[27] There were two ways to address the question of how to establish the microdosimeter response to a known input once on orbit. During the first year of the LRO mission, the galactic cosmic rays were overwhelmingly the dominant environmental input to the dosimeter. Since the GCR environment undergoes small (few percent) variations in intensity on a daily to weekly basis due to changes in the interplanetary medium [e.g., Richardson et al., 1996], we had a time-dependent input that we could use to establish that the dosimeter responded to changes in the GCR intensity. The dosimeter is less responsive to subtle changes to the GCR spectrum as it corresponds to an integral energy measurement, so we focus here on GCR intensity. Thus, we chose to use (1) the contemporaneous 


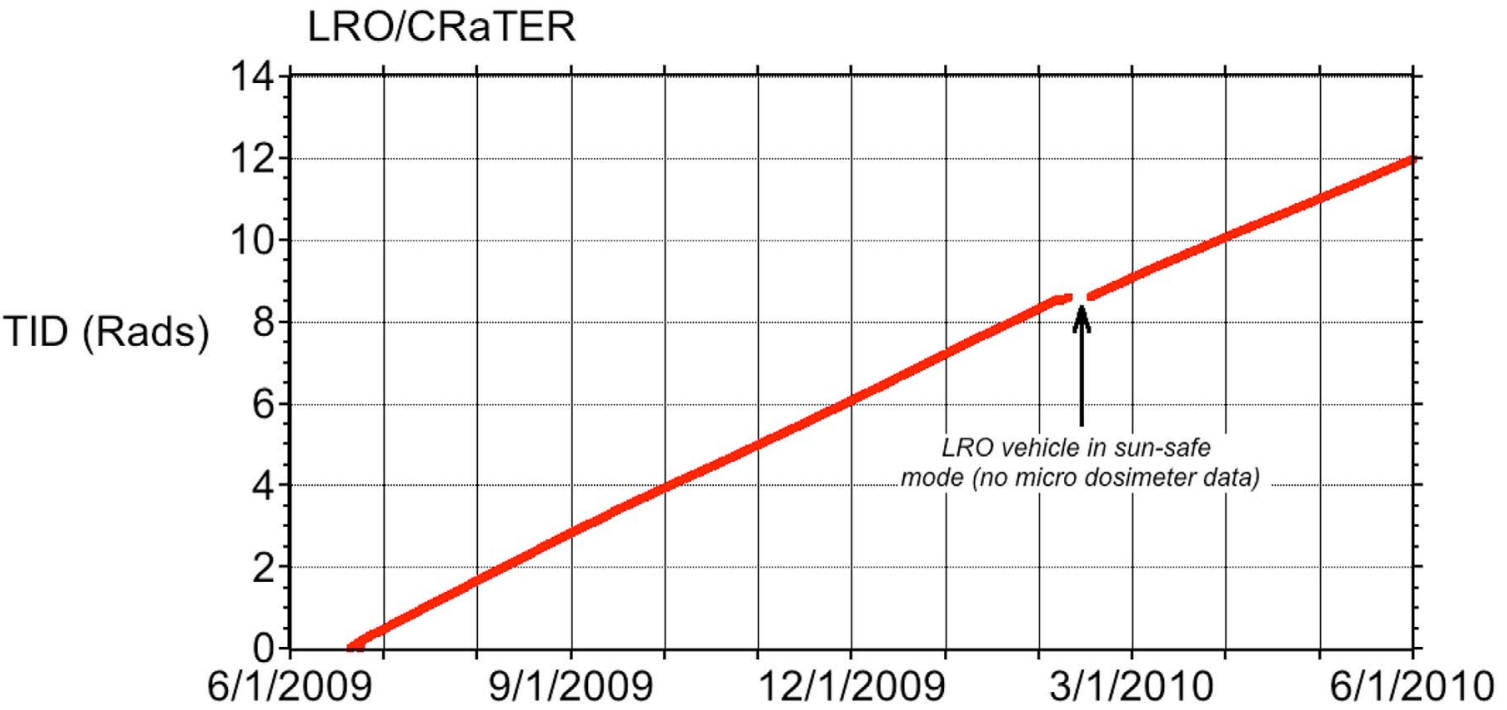

Figure 4. TID as measured with the microdosimeter for the LRO mission from 22 June 2009 through 31 May 2010. The actual TID for the mission to date is approximately 0.2 Rads higher than the result shown on 31 May 2010 because of the $\sim 6$ day power-off interval in mid-February 2010.

GCR measurements from the CRaTER instrument itself and (2) independent measurements of GCR intensity in the near-Earth environment to demonstrate that the dosimeter responded to correctly known inputs. Those changes, taken together with the preflight calibration of the dosimeter, verify its performance as a monitor of TID as discussed below.

[28] Figure 8 compares the dose rate with the threshold response of the outermost zenith-viewing silicon detector (named D1) in the CRaTER telescope with thickness 148 microns, an electronic threshold for ionizing event detections ("singles") of $110 \mathrm{keV}$ [Spence et al., 2010], and zenith shielding of 32 mils aluminum. The outer housing and threshold of D1 combined to yield a response to protons with incident energies from $\sim 12 \mathrm{MeV}$ to over $1 \mathrm{GeV}$. Given its thickness, threshold, and location, the D1 singles rate should have been a reasonable proxy for the environmental input to the microdosimeter. For Figure 8 we calculated daily averages for the time interval of this study without selections on altitude or LRO attitude (the short-term and infrequent changes of LRO attitude away from nominal attitude had no significant effect on these daily averages). The only days excluded from Figure 8 were the 13 days during which we tested changes to the lower-level discriminators of the CRaTER detectors. Figure 8a shows the highly linear relationship between the

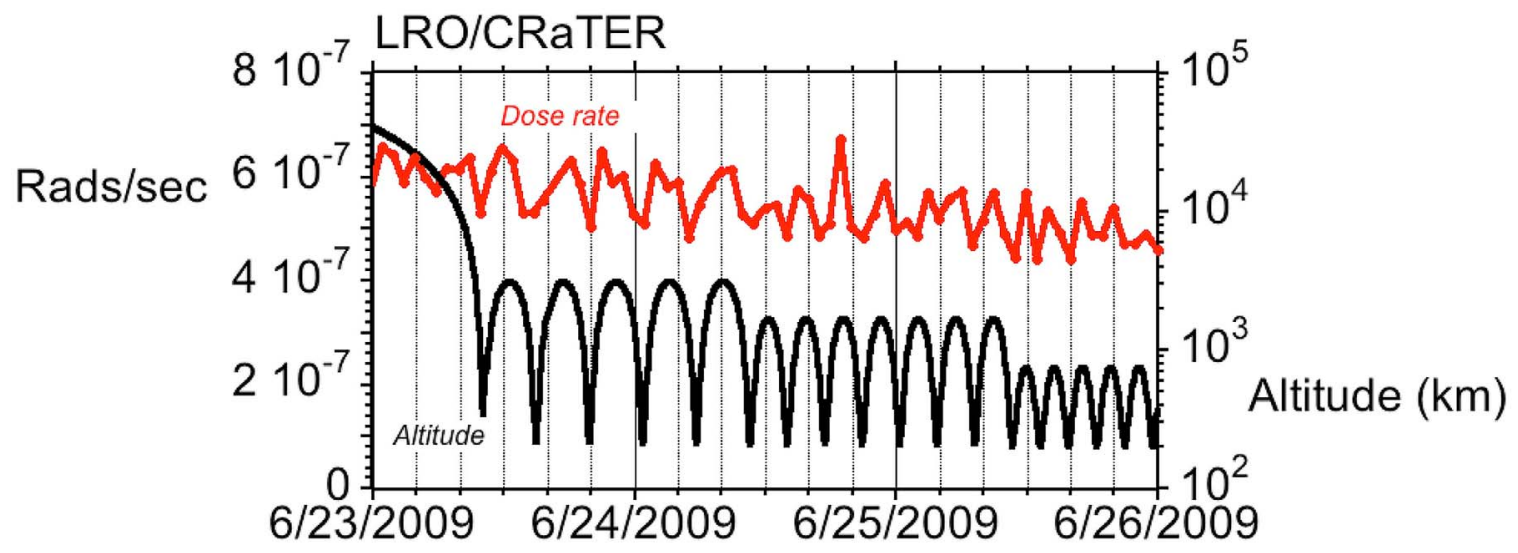

Figure 5. One hour averaged dose rate (Rads per second; red trace) versus time for the first 17 lunar orbits of the LRO mission. The black trace is the LRO altitude above the Moon ( $\mathrm{km}$; Moon principal axis coordinates). There was a clear altitude dependence to the dose rate during these eccentric orbits. 


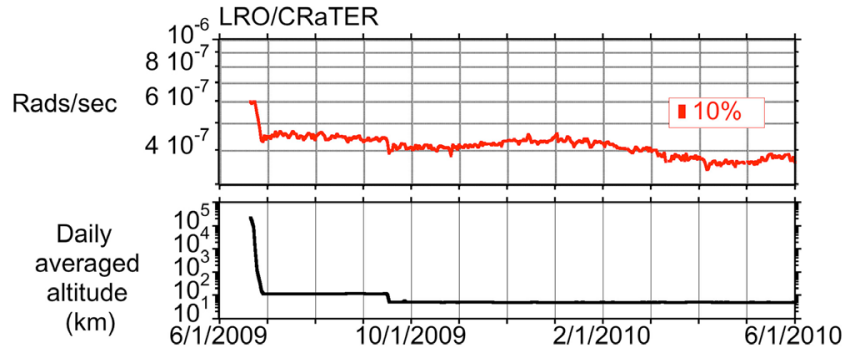

Figure 6. (top) Daily averaged dose rate versus time with (bottom) the daily averaged LRO altitude for reference.

D1 response and the microdosimeter with less than a few percent scatter between them. We found a similar good correlation between the dose rate and the other CRaTER detectors, including the 1000 micron detectors used for lowLET measurements. Using the fit to scale the D1 rate in Figure $8 \mathrm{~b}$ shows that indeed the time histories of the two measurements were nearly identical.

[29] The second test of dosimeter response to an independent measure of GCR uses GCR intensity changes as measured at the first Lagrangian point with the ACE/SIS instrument [Stone et al., 1998]. We chose the corrected ACE/ SIS $>10 \mathrm{MeV}$ proton flux (R. Mewaldt, personal communication, 2010) and normalized both the proton flux and the microdosimeter to their values on 17 September 2009 in Figure 10; this was the first day that LRO was placed into its nominal mission orbit of $\sim 50 \mathrm{~km}$ altitude, thus minimizing the altitude dependence in the dose rate. The comparison required daily averages because of the equivalent low count rate of the dosimeter. While the day-to-day variations in the ACE proton flux and the dose did not track each other as closely as the CRaTER dosimeter and D1 rates in Figure 8, Figure 9 shows that the relative changes in the dose environment and proton flux did indeed track each other, with a rise from September 2009 to January 2010 and Forbush decreases in February, March, and April 2010. In particular, note the $\sim 10 \%$ decrease in both from September 2009 to midMarch 2010. We also note the lack of measurable protons above $10 \mathrm{MeV}$ from solar energetic particle events in this interval, which would have appeared as shorterterm spikes in intensity.

[30] We compared the interplanetary dose rate from LRO with contemporaneous measurements, historic measurements, and model calculations in Figure 10 to assess whether the dose rate was in agreement with expected interplanetary levels. The LRO data in Figure 10 were taken during the cruise of LRO to lunar orbit and thus the lunar obscuration discussed above was not a factor. The other contemporaneous measurements (from the CRaTER detectors and a NASA/TWINS-ES dosimeter) were from the same time period of 19 to 23 June 2009 .

[31] We used a pair of CRaTER detectors to calculate the dose rate in CRaTER. Spence et al. [2010] describe in more detail the selection of detector thickness to cover low-LET and high-LET events. We combined the detailed LET spectra measured in the CRaTER D1-D2 detector pair that faces the zenith. Because CRaTER measures the LET for each valid coincidence event, we were able to integrate the LET spectrum from $\sim 0.1$ to $2000 \mathrm{keV} /$ micron in order to derive the total dose. The statistical uncertainty of the CRaTER result is negligible because of the large number

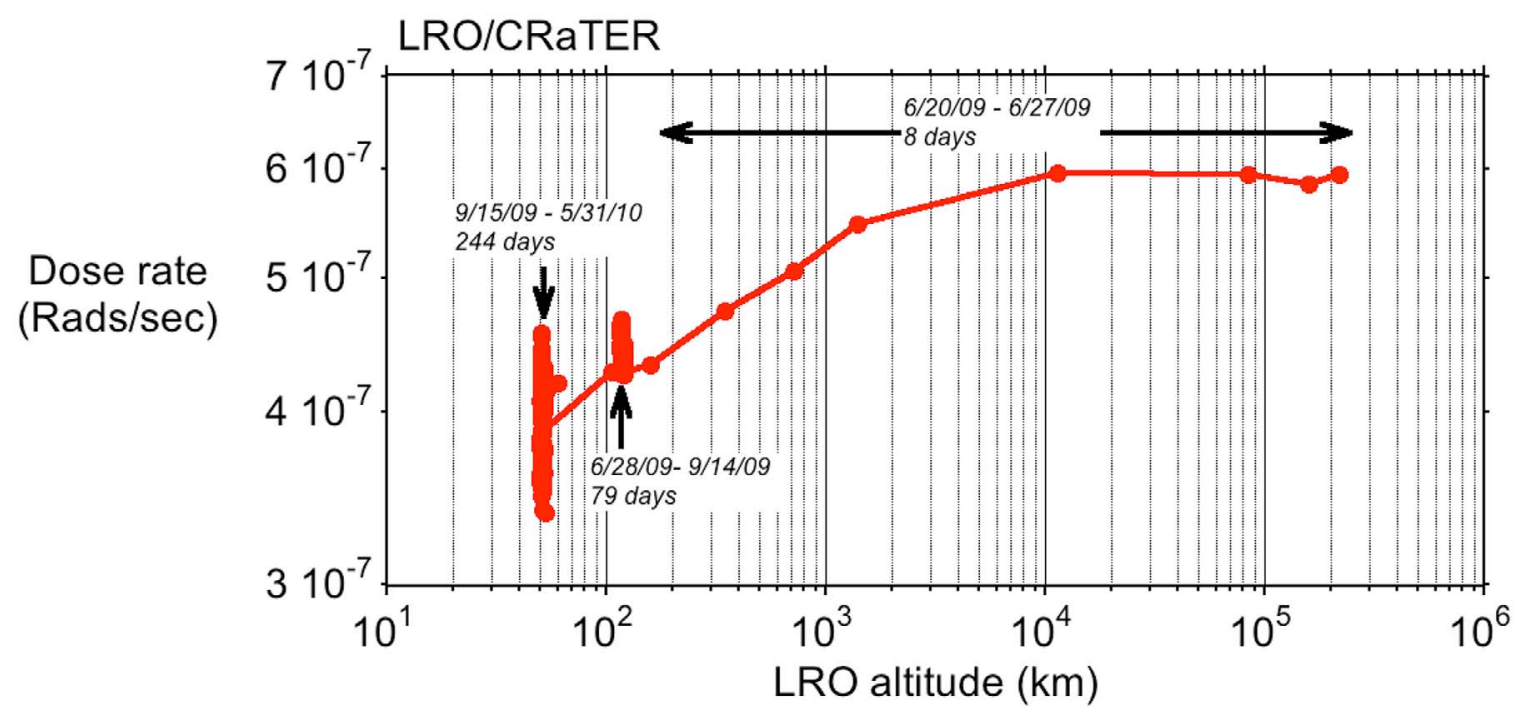

Figure 7. Daily averaged dose rate versus altitude for the study time period. The concentration of data at average altitudes of $\sim 50$ and $210 \mathrm{~km}$ reflects the longer residence times of LRO at those altitudes, and thus the larger scatter of the dose rate at the lower altitudes reflects the time dependence of the GCR. 

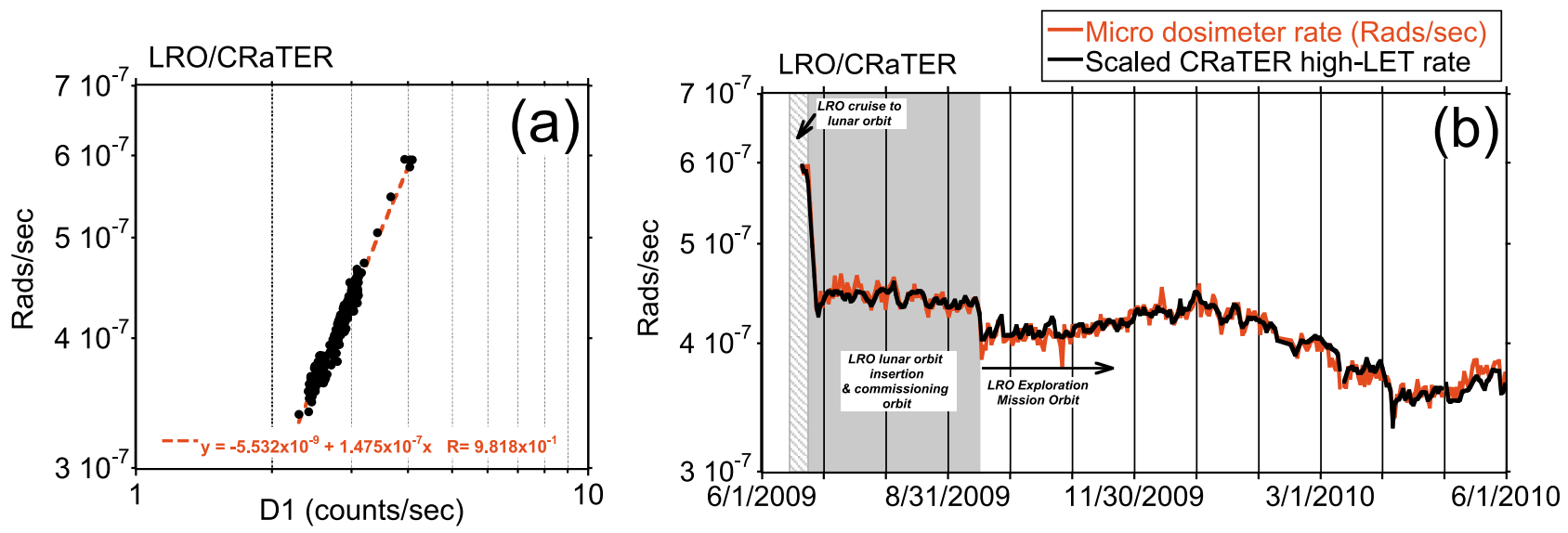

Figure 8. (a) Correlation between the daily averaged microdosimeter dose rate and a high-LET CRaTER detector rate (labeled D1) for the time period of this study; (b) time histories of the dose rate and the D1 response, scaled to a dose rate using the linear conversion in Figure 8a. The LRO daily averages shown here included all CRaTER attitudes relative to the Moon. The shaded regions indicate the LRO transit to the Moon and the LRO commissioning intervals, showing abrupt decreases in the dose rate due to the lunar shadowing of the GCR.

of GCR events collected during one day. Note that the microdosimeter threshold LET is slightly higher $(0.4 \mathrm{keV} /$ micron) than the CRaTER threshold, but nonetheless includes the peak of the minimum-ionizing protons.

[32] The NASA/TWINS-ES dosimeter (J. Clemmons, personal communication, 2010) measures dose in a silicon test mass in a method similar to that employed in the microdosimeter. However, the main physical difference is that the TWINS-ES silicon detector has an aluminum hemispherical shield of 32 mils thickness as an approximation to the dose at the center of a sphere. To derive the interplanetary dose from this device, we selected mea- surements at high-altitude portions of the TWINS orbit that had no contributions from trapped magnetospheric particles. Counting statistics in this device yielded the uncertainty shown in Figure 10. We also show Apollo 16 and Apollo 17 dose rates from the Van Allen Belt Dosimeter that was housed within the Apollo Command Module during similar solar minimum conditions. We note that these Apollo measurements (and all the dose rates shown in Figure 10) are of absorbed dose in Silicon.

[33] Figure 10 (top) contains model calculations of free-space GCR dose rates from the CREME-96 model (https://creme-mc.isde.vanderbilt.edu/CREME-MC), from

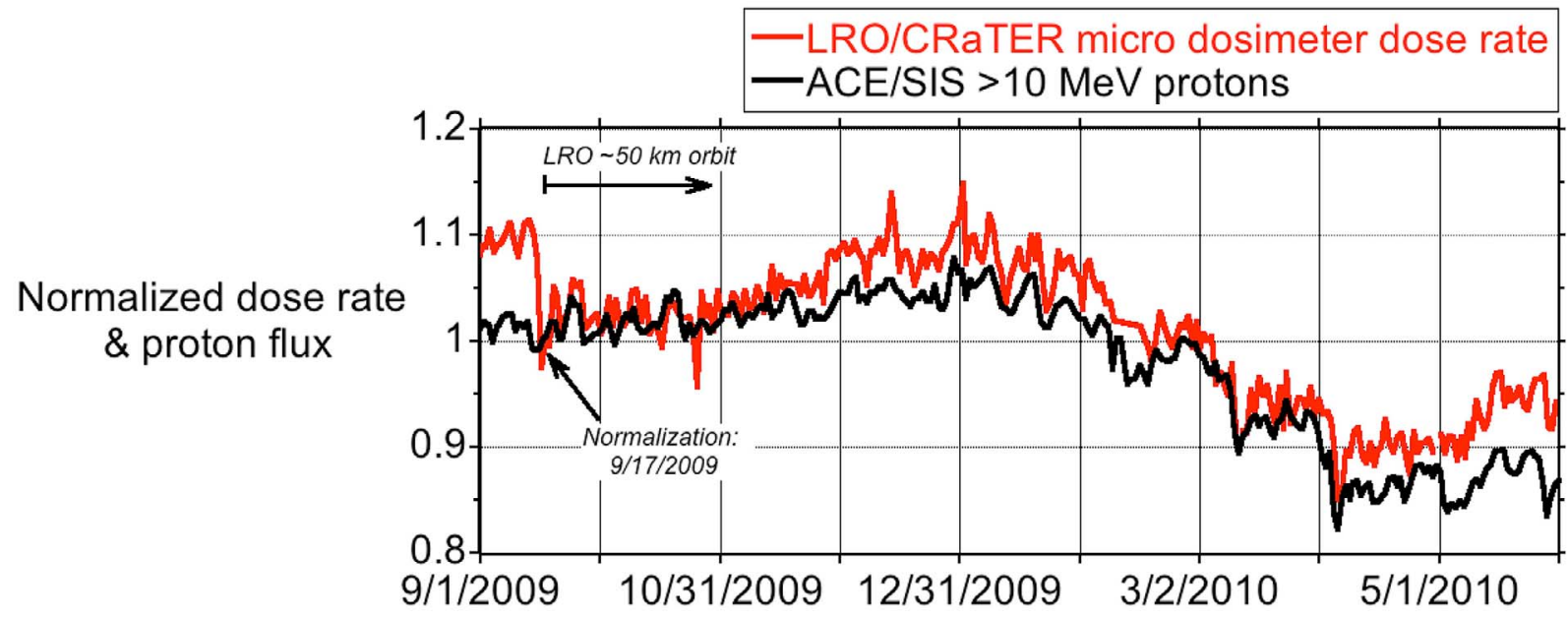

Figure 9. Comparison of the microdosimeter dose rate and the interplanetary proton flux above $10 \mathrm{MeV}$ as measured at ACE. The normalization for both parameters on 17 September 2009 coincided with the first day of the LRO mission orbit with nominal $50 \mathrm{~km}$ altitude. 


\title{
Model calculations
}

\author{
Apollo 16 \& 17 \\ solar minimum
}

\section{Contemporaneous measurements 20 June 2009}

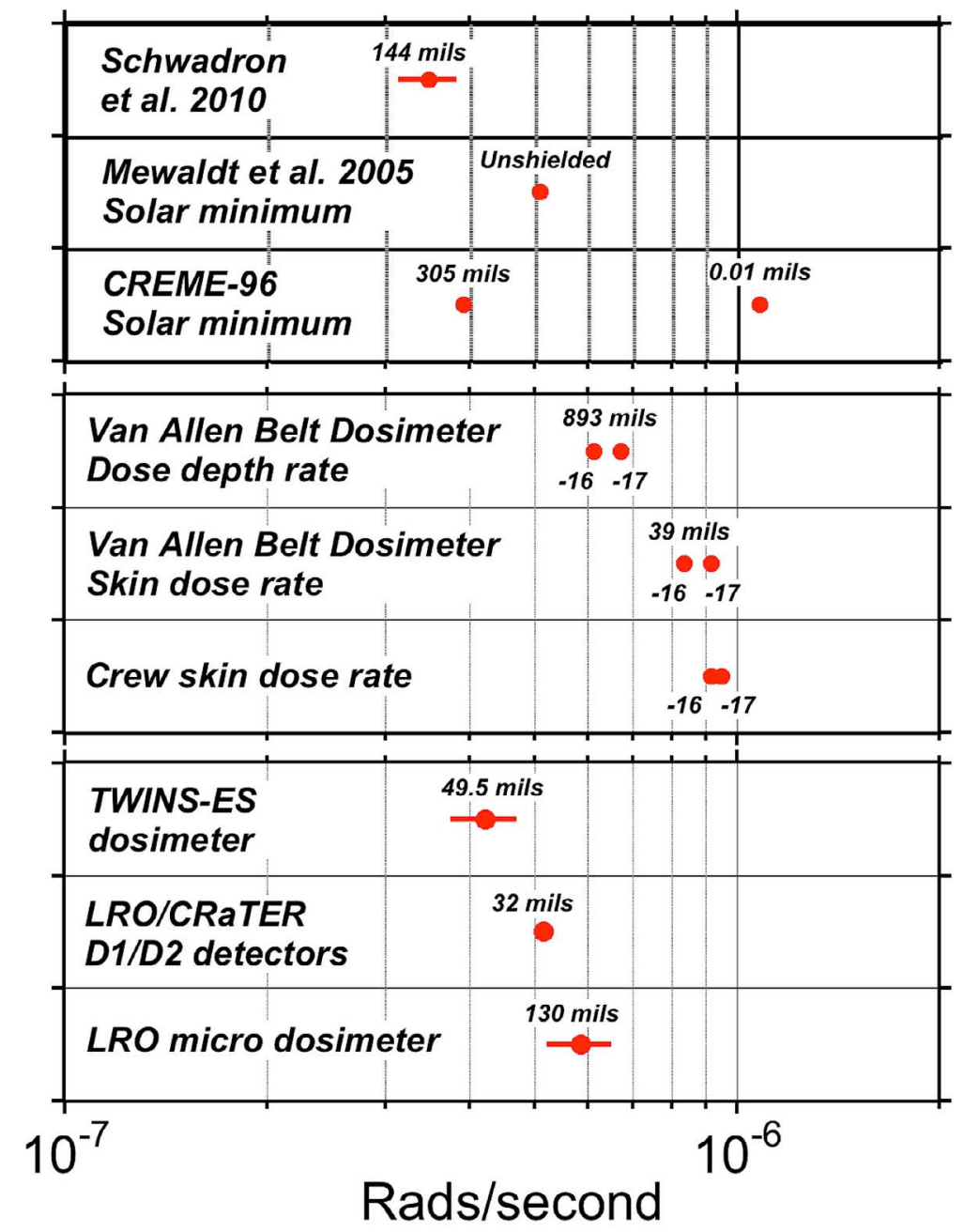

Figure 10. Comparison of (bottom) recent interplanetary dose rate measurements with (middle) historic measurements from Apollo 16 and Apollo 17 and (top) recent model calculations. All panels refer to the GCR dose rate in interplanetary space during solar minimum. See text for details.

a recent calculation of Schwadron et al. [2010] who used the HZETRN model with a Badhwar-O'Neill GCR environment applied to 2009, and from the solar minimum calculation of Mewaldt et al. [2005].

\section{Discussion}

[34] The main requirements for the microdosimeter design have been satisfied: small resource requirements (mass, power, volume, and telemetry) and accurate dose measurements. The LRO radiation environment during the time interval of this study did not include any extremely intense solar particle events, offering an opportunity to test the dosimeter response to the more slowly changing GCR input. Because the GCR dose rate was low (less than 1 micro-Rad per second) compared to typical low-Earthorbit applications and typical solar particle events, these initial results from LRO provided an opportunity to test the dosimeter performance in an environment that was most difficult to measure. In this section, we will discuss the TID observation in the context of other missions, and some details of the dose rate shown above.

[35] For this study period, the GCR was the dominant contributor to the TID and the results to date were two orders of magnitude lower than what was specified for the first year of the LRO mission. The expectation that solar activity would have been halfway to its maximum in solar cycle 24 by the time of LRO launch led to the specification of $\sim 4.6$ kRads under 100 mils aluminum after the first year of the mission (LRO specification 431-SPEC-000020, 2005), in stark contrast to the trifling 12.2 Rads observed.

[36] Also, comparing to model estimates of solar minimum interplanetary dose from GCR, we found that the 
dose rate was $30 \%$ higher than that predicted using the dosimeter's minimum shielding in the CREME-96 model. Indeed, one would have expected a measured dose rate that was $20-30 \%$ higher than CREME-96 solely on the basis of the near-record low solar modulation of GCR at the time of LRO launch (e.g., R. A. Mewaldt et al., 2009; available at http://www.srl.caltech.edu/ACE/ACENews/ ACENews122.html). However, the lack of additional dose sources in the CREME-96 model (no tracking of target fragments for example) would underestimate the predicted dose rate as well, although the detailed assessment of this or other transport codes is outside the scope of this dosimetry analysis. If the low level of solar modulation in June 2009 had been accounted for, we might expect that the calculation of Mewaldt et al. [2005] would be $20 \%$ higher than shown. Changes of this magnitude would place the Mewaldt et al. [2005] result in agreement with the TWINS-ES and CRaTER measurements once some shielding was factored into the calculation.

[37] The Apollo dosimetry was from a previous solar cycle, so one might expect that the different GCR populations could contribute to the dispersion in the measurements. We chose measurements from Apollo 16 and Apollo 17 since those missions also occurred during solar minimum. However, those measurements have likely contributions from trapped particles in the Earth's magnetosphere. This aspect and the uncertainties in exact instrument responses make it unclear how far to pursue an intercalibration between the Apollo and the modern results. Nonetheless, we were encouraged to see the Apollo results yielded similar solar minimum dose rates and therefore that those data may be useful for future analyses of the TID environment on lunar excursions. We intend to report on more details of the Apollo data sets in a separate study (M. Golightly, personal communication, 2010).

[38] We were able to establish that the microdosimeter dose rate, while low compared to other near-Earth environments, nonetheless agreed well with other measurements of the GCR input. The relative changes in the dosimeter rate and the $>10 \mathrm{MeV}$ GCR proton measurements tracked each other to within a few percent; larger $\sim 5 \%$ differences appeared for a few months of the study period (2009 December-2010 January and 2010 AprilMay), possibly originating in changes to the GCR energy spectrum. Even though the microdosimeter was not designed for science, the fact that it measured 10-20\% changes in the low-energy GCR due to solar modulation in agreement with the modulation seen at the NASA ACE spacecraft was a sufficient confirmation of its ability to accurately monitor even relatively small changes in the GCR environment.

\section{Summary and Conclusions}

[39] The microdosimeter housed within the LRO/ CRaTER instrument has sampled the solar minimum GCR environment between the Earth and Moon and at various altitudes in lunar orbit. The microdosimeter's high resolution ( 13 micro-Rads) allowed us to calculate GCR dose rates on the order of micro-Rads per second and to explore how the dose rate compared to simultaneous CRaTER measurements of the GCR environment and other near-Earth measurements. The dosimeter closely followed other contemporaneous monitors of the GCR environment, and in the case of the silicon detectors in the CRaTER instrument, the linear correlation coefficient between dose rate and threshold counts was $>0.92$ on the timescale of one day averages.

[40] We presented three contemporaneous measurements of the TID rate owing to GCR in interplanetary space. Using different techniques, they were all within $20 \%$ of $\sim 0.5$ micro-Rads per second for one particular day in June 2009. The ionizing environment in solar particle events that are certain to occur as the solar cycle progresses will provide additional tests of these measurement techniques and TID models. Taken together, the results to date show that this $\sim 20 \mathrm{~g}$ dosimeter provides accurate and direct monitoring of the TID environment. We thus envision its use in future applications because of its accuracy and its relatively minor requirements for operation.

[41] Acknowledgments. We acknowledge the efforts of the CRaTER instrument team in developing and fielding the instrument hardware, as well as the LRO project team at NASA GSFC for the success of the LRO mission.

\section{References}

Blake, J. B., D. N. Baker, N. Turner, K. W. Ogilvie, and R. P. Lepping (1997), Correlation of changes in the outer-zone relativistic-electron population with upstream solar wind and magnetic field measurements, Geophys. Res. Lett., 24, 927-929, doi:10.1029/97GL00859.

Case, A. W., H. E. Spence, M. J. Golightly, J. C. Kasper, J. B. Blake, J. E. Mazur, L. W. Townsend, and C. J. Zeitlin (2010), GCR access to the Moon as measured by the CRaTER instrument on LRO, Geophys. Res. Lett., 37, L19101, doi:10.1029/2010GL045118.

Cayton, T. E., D. M. Drake, K. M. Spencer, M. Herrin, T. J. Wehner, and R. C. Reedy (1998), Description of the BDD-IIR: Electron and proton sensors on the GPS, Rep. LA-UR-98-1162, Los Alamos Natl. Lab., N. M.

Cucinotta, F., M. Y. Kim, and L. Ren (2005), Managing lunar and Mars mission radiation risks part 1: Cancer risks, uncertainties, and shielding effectiveness, NASA Tech. Publ., NASA/TP-2005-213164, doi:10.1109/TNS.2008.2011485.

Fennell, J. F., J. B. Blake, D. Heynderickx, and N. Crosby (2003), HEO observations of the radiation belt electron fluxes: Comparison with model predictions and a source for model updates, Eos Trans. AGU, 84(46), Fall Meet. Suppl., Abstract SH52A-05.

Freden, S. C., J. B. Blake, and G. A. Paulikas (1965), Spatial variation of the inner zone trapped proton spectrum, J. Geophys. Res., 70, 3113-3116, doi:10.1029/JZ070i013p03113.

Lin, R. P. (1968), Observations of lunar shadowing of energetic particles, J. Geophys. Res., 73, 3066-3071, doi:10.1029/JA073i009p03066.

Mewaldt, R. A., A. J. Davis, W. R. Binns, G. A. de Nolfo, J. S. George, M. H. Israel, R. A. Leske, E. C. Stone, M. E. Wiedenbeck, and T. T. von Rosenvinge (2005), The cosmic ray radiation dose in interplanetary space-Present day and worst-case evaluations, in 29th International Cosmic Ray Conference, Pune, vol. 2, pp. 433-436, Tate Inst. of Fundam. Res., Mumbai, India.

O’Brien, T. P., J. E. Mazur, G. Ginet, and G. Reeves (2008), On-board space environment sensors: Explanations and recommendations, Rep. ATR-2008(8073)-5, Aerosp. Corp., Los Angeles, Calif. 
Pease, R. L., R. D. Schrimpf, and D. M. Fleetwood (2009), ELDRS in bipolar linear circuits: A review, IEEE Trans. Nucl. Sci., 56(4), 1894-1908, doi:10.1109/TNS.2008.2011485.

Richardson, I. G., G. Wibberenz, and H. V. Cane (1996), The relationship between recurring cosmic ray depressions and corotating solar wind streams at $\leq 1$ AU: IMP 8 and Helios 1 and 2 anticoincidence guard rate observations, J. Geophys. Res., 101, 13,483-13,496, doi:10.1029/96JA00547.

Schwadron, N. A., A. J. Boyd, K. Kozarev, M. Golightly, H. Spence, L. W. Townsend, and M. Owens (2010), Galactic cosmic ray radiation hazard in the unusual extended solar minimum between solar cycles 23 and 24, Space Weather, 8, S00E04, doi:10.1029/ 2010 SW000567.

Spence, H. E., et al. (2010), CRaTER: The Cosmic Ray Telescope for the Effects of Radiation Experiment on the Lunar Reconnaissance Orbiter Mission, Space Sci. Rev., 150, 243-284, doi:10.1007/s11214009-9584-8.

Stone, E. C., et al. (1998), The Solar Isotope Spectrometer for the Advanced Composition Explorer, Space Sci. Rev., 86, 357-408, doi:10.1023/A:1005027929871.
Stuckey, W., and M. J. Meshishnek (2003), Ground testing of spacecraft materials, in Crosslink, pp. 36-41, Aerosp. Corp., Los Angeles, Calif.

Vondrak, R., J. Keller, G. Chin, and J. Garvin (2010), Lunar Reconnaissance Orbiter (LRO): Observations for lunar exploration and science, Space Sci. Rev., 150, 7-22, doi:10.1007/s11214-010-9631-5.

J. B. Blake, W. R. Crain, M. D. Looper, and D. J. Mabry, Aerospace Corporation, 2310 E. El Segundo Blvd., El Segundo, CA 90245, USA A. W. Case and J. C. Kasper, Harvard-Smithsonian Center for Astrophysics, 60 Garden St., Cambridge, MA 02138, USA.

M. J. Golightly and H. E. Spence, Institute for the Study of Earth, Oceans, and Space, University of New Hampshire, 8 College Rd., Durham, NH 03824-3525, USA.

J. E. Mazur, Aerospace Corporation, 15049 Conference Center Dr. Chantilly, VA 20151, USA. (joseph.mazur@aero.org) 